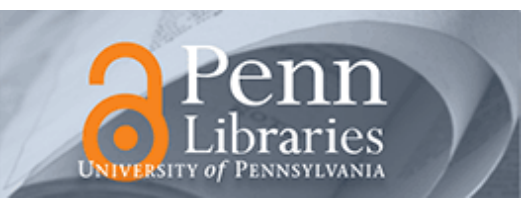

University of Pennsylvania

ScholarlyCommons

March 2008

\title{
A fair scheduling policy for wireless channels with intermittent connectivity
}

\author{
A. Aram \\ University of Pennsylvania \\ M.H. R. Kzouhani \\ University of Pennsylvania \\ Saswati Sarkar \\ University of Pennsylvania, swati@seas.upenn.edu \\ L. Tassiulas \\ University of Thessaly
}

Follow this and additional works at: https://repository.upenn.edu/ese_papers

\section{Recommended Citation}

A. Aram, M.H. R. Kzouhani, Saswati Sarkar, and L. Tassiulas, "A fair scheduling policy for wireless channels with intermittent connectivity", . March 2008.

Copyright 2008 IEEE. Reprinted from 42nd Annual Conference on Computer Sciences and Systems, CISS 2008, March 2008, pages 503-508.

This material is posted here with permission of the IEEE. Such permission of the IEEE does not in any way imply IEEE endorsement of any of the University of Pennsylvania's products or services. Internal or personal use of this material is permitted. However, permission to reprint/republish this material for advertising or promotional purposes or for creating new collective works for resale or redistribution must be obtained from the IEEE by writing to pubs-permissions@ieee.org. By choosing to view this document, you agree to all provisions of the copyright laws protecting it.

This paper is posted at ScholarlyCommons. https://repository.upenn.edu/ese_papers/445

For more information, please contact repository@pobox.upenn.edu. 


\title{
A fair scheduling policy for wireless channels with intermittent connectivity
}

\begin{abstract}
We consider a system of parallel queues with the constraint that only one queue can be served at a time. Each queue can be served through a wireless channel with intermittent connectivity. We propose a policy which serves the longest connected queue when the lengths of all connected are below a given threshold, and serves the connected queue that exceeds the threshold and has received the least service so far, otherwise. We prove that this simple policy (a) maximizes the aggregate service rate of all queues (b) maximizes the minimum service rate attained by any queue and (c) attains the stability region of the network.

Comments

Copyright 2008 IEEE. Reprinted from 42nd Annual Conference on Computer Sciences and Systems, CISS 2008, March 2008, pages 503-508.

This material is posted here with permission of the IEEE. Such permission of the IEEE does not in any way imply IEEE endorsement of any of the University of Pennsylvania's products or services. Internal or personal use of this material is permitted. However, permission to reprint/republish this material for advertising or promotional purposes or for creating new collective works for resale or redistribution must be obtained from the IEEE by writing to pubs-permissions@ieee.org. By choosing to view this document, you agree to all provisions of the copyright laws protecting it.
\end{abstract}




\title{
A Fair Scheduling Policy for Wireless Channels with Intermittent Connectivity
}

\author{
A. Aaram M.H.R. Khouzani, S. Sarkar, L. Tassiulas
}

\begin{abstract}
We consider a system of parallel queues with the constraint that only one queue can be served at a time. Each queue can be served through a wireless channel with intermittent connectivity. We propose a policy which serves the longest connected queue when the lengths of all connected are below a given threshold, and serves the connected queue that exceeds the threshold and has received the least service so far, otherwise. We prove that this simple policy (a) maximizes the aggregate service rate of all queues (b) maximizes the minimum service rate attained by any queue and (c) attains the stability region of the network.
\end{abstract}

\section{INTRODUCTION}

Throughput and fairness guarantees have been obtained in wireless networks owing to several seminal results obtained in the last two decades [8], [4]. Most of these performance guarantees however rely on complex computations. Devices in wireless networks however have limited energy and can therefore only execute low complexity scheduling strategies. Several recent papers have obtained throughput and fairness guarantees using low complexity scheduling strategies[2], [3], [5], [6], [9]. But, most of these papers consider wireless networks where transmission conditions do not change with time. Nevertheless, wireless links often suffer from intermittent connectivity owing to fading and multi-path effects. We seek to attain throughput and fairness guarantees in wireless networks in presence of intermittent connectivity.

We consider a simple topology consisting of one server and $n$ queues. The server can serve at most one queue at a time. The link, or the channel, between the server and any queue is connected only intermittently. A queue can be served only when its channel is connected. Tassiulas [7] proved that scheduling the longest connected queue (LCQ) attains the maximum possible stability region in such networks. But, as the following example demonstrates, LCQ can treat some queues unfairly when the arrival rate vector is outside the network stability region. Suppose there are two queues $\left(q_{1}\right.$ and $\left.q_{2}\right)$ and the channels between the queues and the server are always connected. Two packets arrive at $q_{1}$ in each slot and one packet arrives at $q_{2}$ every other slot. Clearly, in this case, LCQ will always serve $q_{1}$ and never serve $q_{2}$, allocating rates of $(1,0)$ to them. Now, consider another policy, the Least Service First or LSF, that serves the non-empty connected queues that has received the least

A. Aaram, M.H.R. Khouzani and S. Sarkar are with the Dept. of Electrical and System Engineering at University of Pennsylania. Their email addresses are aaram@seas.upenn.edu, khouzani@seas.upenn.edu, and swati@seas.upenn.edu. L. Tassiulas is with the Dept. of Computer and Telecommunications Engineering, University of Thessaly, Greece. His email address is leandros@uth.gr. accumulative service from the server. Intuitively, it is fairer than LCQ. For example, in the above specific case, it allocates a rate of 0.5 to each of the queues. Nevertheless, as the following examples demonstrate, LSF is inefficient in that (a) it does not attain the maximum possible stability region, and (b) does not maximize the aggregate service rate irrespective of whether the arrival rate vector is inside or outside the network stability region.

Example 1. Consider two queues $q_{1}$ and $q_{2}$ and let the channel for queue 1 be connected in odd numbered slots, and the channel for queue 2 always be connected. Queue $q_{1}$ receives a packet in every odd slot, and $q_{2}$ receives a packet in slots $3,6, \ldots$ The corresponding arrival rate vector is in the stability region of the network as a policy (say policy $P$ ) that serves $q_{1}$ in odd slots, and $q_{2}$ in slots $3 i+1, i=0,1, \ldots$ stabilizes both queues. $P$ allocates a service rate vector of $(1 / 2,1 / 3)$. LSF serves $q_{2}$ whenever it receives packets, and $q_{1}$ in slots in which $q_{1}$ is connected and $q_{2}$ does not receive a packet. Thus, each queue is served at rate $1 / 3$. Thus, LSF does not stabilize $q_{1}$. Thus, LSF does not attain the stability region of the network, and does not maximize the aggregate service rate. Now suppose that $q_{1}$ has packet arrivals in every slot. Now, note that $q_{1}$ can not be stabilized under any policy as its packet arrival rate exceeds its connectivity rate. Thus, the arrival rate vector is outside the network stability region. The service process under LSF remains the same, and thus LSF serves each queue at rate $1 / 3$. As before, policy $P$ can serve each queue at rate $1 / 2$. thus, $L S F$ does not maximize the aggregate service rate in this case either.

We now explore whether the above policies (LCQ and LSF) can be combined to attain both efficiency and fairness. We propose a policy that serves the (a) longest connected queue while all connected queues are below a certain threshold and (b) the connected queue that has received the least accumulative service so far among all connected queues, otherwise. We prove that this policy (a) maximizes the aggregate service rate of all queues (b) maximizes the service rate of the queue that receives the minimum service rate and (c) maximizes the stability region of the network (i.e., stabilizes the network if the arrival rate vector is in the network stability region). The policy and the proof constitute the main contributions of the paper.

\section{System Model}

We assume that there exists a single server and $n$ queues: $Q=\{1,2, \cdots, n\}$. Time is slotted. The server can serve at most one queue in a given slot, and serves one packet from such a queue in the slot. The link or the channel 
between each queue and the server can either be connected (ON) or disconnected (OFF). For any $Q_{i}, Q_{j} \subseteq Q$ with $Q_{i} \cap Q_{j}=\emptyset$, let $\mu_{Q_{i}, \overline{Q_{j}}}\left(t_{k}, t_{l}\right)$ be the number of slots in the time interval $\left[t_{k}, t_{l}\right]$ during which at least one of the queues in $Q_{i}$ is connected while all of the queues in $Q_{j}$ are disconnected. Packets arrive to a queue at the beginning of a slot and can be transmitted in the same slot. Let $A_{Q_{i}}\left(t_{k}, t_{l}\right)$ be the aggregate number of packets that arrived to queues in $Q_{i} \subseteq Q$ during interval $\left[t_{k}, t_{l}\right]$. A queue can be served only when it is connected and it has a packet to transmit. Let $S_{Q_{i}}\left(t_{k}, t_{l}\right)$ be the total number of packets served from queues in $Q_{i} \subseteq Q$ during interval $\left[t_{k}, t_{l}\right]$.

We assume that queues have unlimited buffer size. Let $X_{i}(t)$ be the number of packets waiting for transmission in queue $i$ at time $t$. We assume that $X_{i}(0)=0$ for all $i$. In each slot $t, X_{i}(t)$ increases by $A_{\{i\}}(t, t)$, and decreases by 1 if queue $i$ is served in $t$. Let $M$ be the maximum amount by which $X_{i}(t)$ can change in one time slot for any $i$.

We assume that the processes $\mu_{Q_{i}, \overline{Q_{j}}}\left(t_{k}, t_{l}\right)$ and $A_{Q_{i}}\left(t_{k}, t_{l}\right)$ are pseudo-deterministic. In other words, there exists numbers $\left\{a_{i}\right\},\left\{\mu\left(Q_{i}, \bar{Q}_{j}\right)\right\}, \Delta$ such that for any $Q_{i}, Q_{j}, t_{k}, t_{l}$ we have:

$$
\begin{aligned}
\left|\mu_{Q_{i}, \overline{Q_{j}}}\left(t_{k}, t_{l}\right)-\mu\left(Q_{i}, \overline{Q_{j}}\right)\left(t_{l}-t_{k}\right)\right| & \leq \Delta \\
\left|A_{Q_{i}}\left(t_{k}, t_{l}\right)-\sum_{j \in Q_{i}} a_{j}\left(t_{l}-t_{k}\right)\right| & \leq \Delta .
\end{aligned}
$$

The numbers $\left\{a_{i}\right\},\left\{\mu\left(Q_{i}, \overline{Q_{j}}\right)\right\}$ are denoted as the arrival rates and connectivity rates respectively, and $\left(a_{1}, \ldots, a_{n}\right)$ is denoted as the arrival rate vector. Clearly, $M=$ $\max \left(\max _{i} a_{i}, \max _{j} \mu\left(q_{j}, \bar{\emptyset}\right)\right)+\Delta$.

We make the following technical assumption.

$$
\mu\left(Q_{i}, \overline{Q_{j}}\right) \neq \sum_{l \in Q_{i}} a_{l}
$$

for all disjoint $Q_{i} \neq \emptyset$ and $Q_{j}$.

A queue $i$ is said to be stable if $\sup _{t} X_{i}(t)<\infty$. The stability of a queue depends on the arrival and connectivity processes and the scheduling policy. We say the arrival rates are inside the stability region of the system iff for some scheduling policy, all queues are stable. A scheduling policy is said to attain the stability region of the system provided it can stabilize the system for any arrival rate vector that is in the stability region of the system.

Lemma 1. Given any set of arrival and connectivity parameters, we can always partition the set of queues into two subsets: Subset $Y$ and subset $Z=Y^{c}$ with the following properties:

$$
\mu\left(Q_{i}, \bar{Z}\right)>\sum_{j \in Q_{i}} a_{j} \quad \text { for all } \emptyset \subset Q_{i} \subseteq Y,
$$

and

$$
\mu\left(Q_{k}, \overline{Z \backslash Q_{k}}\right)<\sum_{j \in Q_{k}} a_{j} \text { for all } \emptyset \subset Q_{k} \subseteq Z .
$$

Moreover, this partitioning is unique.
The proof of Lemma 1 reveals that the pair $(Y, Z)$ where $Y$ is the largest cardinality set that satisfies (4) and $Z=Y^{c}$ constitutes the above unique partition.

The following argument establishes that the arrival rates are not inside the stability region of the system if $Z \neq \emptyset$. Note that this fact is consistent with the result of [7] for a different arrival and connectivity model. From Lemma 1, for any disjoint $\phi \subset Q \subseteq Z$, we have

$$
\mu(Q, \overline{Z \backslash Q})<\sum_{l \in Q_{i}} a_{j}
$$

Thus, from (1) and (2), $\sum_{j \in Z} X_{j}(t) \geq t \sum_{j \in Z}\left(a_{j}-\right.$ $\mu(Z, \bar{\emptyset}))-2 \Delta$. Thus,

$$
\sup _{j \in Z} X_{j}(t)=\infty
$$

\section{Scheduling Policy and Performance Guarantees}

We now present our scheduling policy $f(L)$ which schedules the queues as follows. Let $U(t)$ be the set of queues that are connected in $t$ and have queue lengths greater than or equal to a threshold $L$. If $U(t) \neq \emptyset$, the queue in $U(t)$ that has received the least service so far is served, else, the connected queue with the maximum queue length is served. The policy is parameterized with parameter $L$, and does not require the scheduler to know the arrival and connectivity rates and $\Delta$.

The following theorem shows that for a large enough $L$, the buffer sizes of the queues in $Y$ are upper bounded under $f(L)$. Also, the buffer sizes of the queues in $Z$ are larger than that of those in $Y$ after a finite time. Note that as a special case, this shows that $f(L)$ stabilizes the arrival rates inside the throughput region.

Theorem 1. There exist $D_{0}$ and $L\left(D_{0}\right)$, which depend only on the system parameters, such that for all $L>L\left(D_{0}\right)$, we have the following.

$$
\exists t_{0} \quad \text { s.t }\left\{\begin{array}{l}
\sup _{\substack{j \in Y \\
t \geq t_{0}}} X_{j}(t)<D_{0} \\
\inf _{\substack{j \in Z \\
t \geq t_{0}}} X_{j}(t)>D_{0}
\end{array} .\right.
$$

The proof of the theorem relies on the following lemmas which we prove in the appendix (Lemmas' 3,4 ) and in a technical report [1] (Lemma 2).

Lemma 2. Let $T=D_{0}^{1 / 4}, L>\sqrt{2 D_{0}}+M D_{0}^{1 / 4}$. Then, for all large enough $D_{0}$,

$\sum_{j \in Y} X_{j}^{2}(t+T)-\sum_{j \in Y} X_{j}^{2}(t) \leq 0 \quad$ if $\quad D_{0} \leq \sum_{j \in Y} X_{j}^{2}(t) \leq 2 D_{0}$

The minimum required value of $D_{0}$ for the above lemma to hold depends only on $n, M$ arrival and connectivity rates and not on $t$ or the queue lengths at $t$. 
Lemma 3. For all $D^{\prime} \geq 0$, there exist $L_{0}$, which depends only on $D^{\prime}$ and the arrival and service parameters, such that for all $L>L_{0}$,

$$
\sum_{j \in Z} X_{j}^{-2}\left(t_{0}\right)<\frac{1}{D^{\prime}}
$$

for some finite $t_{0}$.

Lemma 4. For all $\bar{D}>D_{\text {min }}$, there exists $T$ and large enough $L_{\bar{D}}$ such that if $L>L_{\bar{D}, T}$, and for some $t, 1 / \bar{D}<$ $\sum_{j \in Z} X_{j}^{-2}(t) \leq n /(\sqrt{\bar{D}}-M T)^{2}$, we have

$$
\sum_{j \in Z} X_{j}^{-2}(t+T)-\sum_{j \in Z} X_{j}^{-2}(t) \leq 0 .
$$

Here, $D_{\min }$ and $T$ depend only on the parameters of the system, and $L_{\bar{D}}$ depends only on $\bar{D}, T$ and the parameters of the system.

Proof of Theorem 1: We first show that there exists $D^{\prime}$ such that for all $L>\sqrt{D^{\prime}}$,

$$
\sup _{t \geq 0} \sum_{j \in Y} X_{j}^{2}(t) \leq D^{\prime}
$$

We next show that there exists $\hat{D}>D^{\prime}$ such that for a finite $t_{0}$ and $L$ large enough,

$$
\sum_{j \in Z} X_{j}^{-2}(t) \leq 1 / \hat{D} \forall t \geq t_{0}
$$

The Theorem follows.

We now show (8). Let $D_{0}$ satisfy the minimum required value for Lemma 2 to hold, and, in addition, $D_{0}$ be large enough such that

$$
D^{\prime}=2 D_{0} \geq\left(\sqrt{D_{0}}+\sum_{j \in Y}\left(a_{j}\left(D_{0}\right)^{1 / 4}+\Delta\right)\right)^{2} .
$$

Let $T=D_{0}^{1 / 4}, L>\sqrt{2 D_{0}}+M D_{0}^{1 / 4}$.

Now we show by induction that

$$
\sum_{j \in Y} X_{j}^{2}(t+k T) \leq D^{\prime} \quad \forall t \in[0, T], k \geq 0 .
$$

The base case corresponds to $k=0$, and holds since $T=$ $D_{0}^{1 / 4}$ and $X_{j}(t) \leq a_{j} T+\Delta$ for each $j$ and $t \in[0, T]$. Now, let the hypothesis hold for $k=0, \ldots, m$. Consider a $t \in[0, T]$. If $\sum_{j \in Y} X_{j}^{2}(t+m T) \leq D_{0}$, then, clearly, $\sum_{j \in Y} X_{j}^{2}(t+(m+$ 1) $T) \leq D^{\prime}$ since each $X_{j}$ can increase only by $a_{j} T+\Delta$ in $[t+m T, t+(m+1) T]$. If $D_{0} \leq \sum_{j \in Y} X_{j}^{2}(t+k T) \leq$ $2 D_{0}=D^{\prime}$, then by Lemma $2, \sum_{j \in Y} X_{j}^{2}(t+(m+1) T) \leq$ $\sum_{j \in Y} X_{j}^{2}(t+m T) \leq D^{\prime}$.

We now show (9). Select $\bar{D}$ such that $\bar{D} \geq D_{\min }$ in Lemma 4 , and $\hat{D}=\frac{(\sqrt{\bar{D}}-M T)^{2}}{n}>D^{\prime}$. Now, from Lemma 3 , there exists $L_{0}, t_{0}$ such that

$$
\sum_{j \in Z} X_{j}^{-2}\left(t_{0}\right)<1 / \bar{D} \text { for } L>L_{0} .
$$

Select $T$ such that Lemma 4 holds, and let $L_{1}=L_{\bar{D}, T}$ given in Lemma 4 . The result follows if we can show that for all $L>\max \left(L_{0}, L_{1}\right)$,

$$
\sum_{j \in Z} X_{j}^{-2}(t+k T) \leq 1 / \hat{D} \quad \forall t \in\left[t_{0}, t_{0}+T\right], k \geq 0 .
$$

We prove the above using induction on $k$. We first prove the above for $k=0$. Clearly, since $\sum_{j \in Z} X_{j}^{-2}\left(t_{0}\right) \leq 1 / \bar{D}$, then $\sum_{j \in Z} X_{j}^{-2}\left(t_{0}+t\right) \leq 1 / \hat{D}$ for all $t \in[0, T]$. Now, we assume that the above hypothesis holds for $k$, and prove it for $k+1$. Consider some $t \in[0, T]$. If $\sum_{j \in Z} X_{j}^{-2}(t+$ $k T) \leq 1 / \bar{D}$, then, clearly, $\sum_{j \in Z} X_{j}^{-2}(t+(k+1) T) \leq 1 / \hat{D}$. If $1 / \bar{D}<\sum_{j \in Z} X_{j}^{-2}(t+k T) \leq 1 / \hat{D}$, then by Lemma 4 , $\sum_{j \in Z} X_{j}^{-2}(t+(k+1) T) \leq \sum_{j \in Z} X_{j}^{-2}(t+k T) \leq 1 / \hat{D}$. The result follows.

We have already argued that if $Z \neq \emptyset$ then the arrival rates are not inside the stability region of the system. Theorem 1 shows that if $Z=\emptyset$ then $f(L)$ stabilizes the system for all large enough $L$. Thus, for any arrival rate vector that is inside the stability region of the system, $f(L)$ stabilizes the system for all large enough $L$. Thus, this policy attains the stability region of the system. We next use Theorem 1 to prove the following main results of the paper.

Theorem 2. For $L$ large enough, the policy $f(L)$ maximizes both the aggregate and the minimum service rates.

Proof: Let $L$ be such that for some $D>0$ and finite $t_{0}$,

$$
\left\{\begin{array}{l}
\sup _{\substack{j \in Y \\
t \geq t_{0}}} X_{j}(t)<D \\
\inf _{\substack{j \in Z \\
t \geq t_{0}}} X_{j}(t)>D
\end{array} .\right.
$$

From Theorem $1, L, D$, and $t_{0}$ as above exist. Therefore, under $f(L)$, all queues in $Y$ are stable and their lengths are less than that of those in $Z$ after a finite time. As a result, whenever a queue in $Z$ is connected, one of them will be served. This means that $f(L)$ maximizes the aggregate service rate of $Z$. Moreover, since queues in $Y$ are all stable, this subset also receives the maximum service. Therefore, $f(L)$ maximizes the aggregate service rate.

In the following, we proceed to prove the second claim. We use the following assumption without a proof: There exist a service rate for each queue.

Suppose $\left\{q_{1}, q_{2}, \cdots, q_{k}\right\}$ is the set of queues which receive the minimum service rate $r_{1}=r_{2}=\cdots=r_{k}$ under $f(L)$. Also suppose there is a policy which gives rates $r_{1}^{\prime}, r_{2}^{\prime}, \cdots, r_{k}^{\prime}$ to these queues where $r_{i}^{\prime}>r_{i}$ for $i=1, \cdots, k$. So under $f(L), q_{1}, \cdots, q_{k}$ are all unstable. Therefore, after a finite time, their lengths are always greater than $L$. This means that if at least one queue in the set $\left\{q_{1}, q_{2}, \cdots, q_{k}\right\}$ is connected, one of them will receive the service. So the set $\left\{q_{1}, q_{2}, \cdots, q_{k}\right\}$ receives the maximum possible service among all policies. As a result $\sum_{i=1}^{k} r_{i} \geq \sum_{i=1}^{k} r_{i}^{\prime}$, which is a contradiction. Hence, $f(L)$ maximizes the minimum 
service rate.

Putting these together, the theorem follows.

The proof that $f(L)$ maximizes the minimum service rate relies on the assumption that the service rates $\lim _{t \rightarrow \infty} S_{\{j\}}(0, t) / t$ exist for each queue $j$ under $f(L)$. Extensive simulations, which we present in our technical report [1], demonstrate that this is indeed the case.

Another interesting question is whether the above simple policy attains the max-min fair service rate. A service rate allocation is max-min fair if it has the maximum value of the minimum component among all service rate vectors, and subject to the above the maximum value of the second minimum and so on. The following example reveals that this is not true in general.

Example 2. Let $n=3$ and the connectivity and arrival processes constitute mutually independent Bernoulli processes with rates $\mu(\{1\}, \phi)=1 / 7, \mu(\{2\}, \phi)=$ $0.3, \mu(\{3\}, \phi)=5 / 6, a_{1}=0.1, a_{2}=0.7, a_{3}=0.7$. Simulation reveals that our policy provides service rates of $(0.1,0.27,0.53)$.

Nevertheless, our extensive simulations also indicate that in most of the cases, the achieved service rates are very close to the max-min fair rates.

\section{Appendix}

\section{A. Proof of Lemma 1} $Q \subseteq Z$

Proof: First, we claim that if for some $Z$ and $\emptyset \subset$

$$
\mu(Q, \overline{Z \backslash Q})>\sum_{j \in Q} a_{j},
$$

then there exist $\emptyset \subset \hat{Q} \subseteq Q$ such that

$$
\mu\left(Q_{i}, \overline{Z \backslash \hat{Q}}\right)>\sum_{j \in Q_{i}} a_{j} \text { for all } \emptyset \subset Q_{i} \subseteq \hat{Q} .
$$

We prove this claim by induction on $|Q|$. For $|Q|=1$, the claim easily follows. Now suppose the claim is true for $|Q|=k-1$, we show it is also true for $|Q|=k$. If (11) holds for $Q=\hat{Q}$, the claim follows. So, suppose there exist $Q_{i} \subset Q$ such that $\mu\left(Q_{i}, \overline{Z \backslash Q}\right)<\sum_{j \in Q_{i}} a_{j}$. Consider the subset $Q \backslash Q_{i}$. It is clear that

$$
\begin{array}{r}
\mu\left(Q_{i}, \overline{Z \backslash Q}\right)+\mu\left(Q \backslash Q_{i}, \overline{Z \backslash\left(Q \backslash Q_{i}\right)}\right) \\
=\mu(Q, \overline{Z \backslash Q}),
\end{array}
$$

which, together with (3), (10) and the fact that $\mu\left(Q_{i}, \overline{Z \backslash Q_{i}}\right)<\sum_{j \in Q_{i}} a_{j}$, gives

$$
\mu\left(Q \backslash Q_{i}, \overline{Z \backslash\left(Q \backslash Q_{i}\right)}\right)>\sum_{j \in Q \backslash Q_{i}} a_{j} .
$$

Now, $Q \backslash Q_{i}$ satisfies (10) and $\left|Q \backslash Q_{i}\right|<|Q|=k$. Therefore there exist $\hat{Q} \subseteq\left(Q \backslash Q_{i}\right)$ that satisfies (11) and the claim follows.

Now we proceed to prove the lemma. Let $Y_{1}$ be the largest cardinality set (which can be an empty set) that satisfies
(4). We show that $Z_{1}=Y_{1}^{c}$. satisfies (5). Then the lemma follows for $Y=Y_{1}$ and $Z=Z_{1}$. Let $Z_{1}$ not satisfy (5). Then, for some $\emptyset \subset Q \subseteq Z_{1}$

$$
\mu\left(Q, \overline{Z_{1} \backslash Q}\right)>\sum_{j \in Q} a_{j}
$$

By the claim proved above, there exist $\emptyset \subset \hat{Q} \subseteq Q$ such that

$$
\mu\left(Q_{i}, \overline{Z_{1} \backslash \hat{Q}}\right)>\sum_{j \in Q_{i}} a_{j} \quad \text { for all } \quad Q_{i} \subseteq \hat{Q} .
$$

Now consider $Y_{2}=Y_{1} \cup \hat{Q}$. Clearly, for all $\emptyset \subset Q_{i} \subseteq Y_{1}$, $\mu\left(Q_{i}, \overline{Z_{1} \backslash \hat{Q}}\right) \geq \mu\left(Q_{i}, \overline{Z_{1}}\right)$. Also, for all $\emptyset \subset Q_{i} \subseteq Y_{1}$ and $\emptyset \subset Q_{j} \subseteq \hat{Q}$,

$$
\begin{aligned}
\mu\left(Q_{i} \cup Q_{j}, \overline{Z_{1} \backslash \hat{Q}}\right) & =\mu\left(Q_{i}, \overline{Z_{1} \backslash\left(\hat{Q} \backslash Q_{j}\right)}\right)+\mu\left(Q_{j}, \overline{Z_{1} \backslash \hat{Q}}\right) \\
& \geq \mu\left(Q_{i}, \overline{Z_{1}}\right)+\mu\left(Q_{j}, \overline{Z_{1} \backslash \hat{Q}}\right) .
\end{aligned}
$$

Thus, from (12) and since $Y_{1}$ satisfies (4), $Y_{2}$ satisfies (4). But, $\left|Y_{2}\right|>\left|Y_{1}\right|$ which is a contradiction.

Now we show the uniqueness of $Y$ and $Z$. Suppose there are two different partitioning pairs $\left(Y_{1}, Z_{1}\right)$ and $\left(Y_{2}, Z_{2}\right)$ that satisfy (4) and (5). Now consider the subset $Z_{2} \backslash Z_{1} \neq$ $\emptyset$. From $Z_{2} \backslash Z_{1} \subseteq Y_{1}$ and (4) we have

$$
\mu\left(Z_{2} \backslash Z_{1}, \overline{Z_{1}}\right)>\sum_{j \in Z_{2} \backslash Z_{1}} a_{j},
$$

while from $Z_{2} \backslash Z_{1} \subseteq Z_{2}$ and (5) we have

$$
\begin{aligned}
\mu\left(Z_{2} \backslash Z_{1}, \overline{Z_{2} \backslash\left(Z_{2} \backslash Z_{1}\right)}\right) & =\mu\left(Z_{2} \backslash Z_{1}, \overline{Z_{2} \cap Z_{1}}\right) \\
& <\sum_{j \in Z_{2} \backslash Z_{1}} a_{j},
\end{aligned}
$$

It is now clear that (13) and (14) cannot be true at the same time which contradicts the assumption of the existence of different partitioning pairs.

\section{B. Proof of Lemma 3}

Proof: It is sufficient to show that for some finite $t_{0}$, $X_{j}\left(t_{0}\right)>\sqrt{n D^{\prime}}$ for all $j$ in $Z$. We prove the lemma by induction. From (7), for any $D^{\prime} \geq 0$, there exist $L_{0}$ such that for all $L>L_{0}, X_{j}\left(t_{0}\right)>D^{\prime}$ for at least one $j$ in $Z$ and a finite time $t_{0}$. Now, suppose for all $\bar{D}$, there exists $t_{0}, L_{0}$ such that for all $L>L_{0}, X_{j}\left(t_{0}\right)>\bar{D}$ for at least $k$ queues in $Z$, where $k \geq 1$. We will show that for all $D^{\prime}$ there exists $t_{1}, L_{0}$ such that for all $L>L_{0}, X_{j}\left(t_{1}\right)>D^{\prime}$ for at least $k+1$ queues.

Let $r=\min _{\emptyset \subset Q \subseteq Z}\left(\sum_{i \in Q} a_{i}-\mu(Q, \overline{Z \backslash Q})\right)$. From (6), $r>0$. Let $\bar{D}=(1+(n M / r)) D^{\prime}+(M / r) 2 \Delta$. By induction hypothesis, there exists there exists $t_{0}, L_{0}>\bar{D}$ such that for all $L>L_{0}, X_{j}\left(t_{0}\right)>\bar{D}$ for at least $k$ queues in $Z$. Let $B$ be the set of those $k$ queues. If $X_{j}\left(t_{0}\right)>D^{\prime}$ for any $j \in Z \backslash B$, since $\bar{D}>D^{\prime}, X_{j}\left(t_{0}\right)>D^{\prime}$ for at least $k+1$ queue in $Z$ and the claim follows with $t_{1}=t_{0}$. Now consider the case that $X_{j}\left(t_{0}\right)<D^{\prime}$ for all $j \in Z \backslash B$. 
Let $T=(n / r) D^{\prime}+(2 \Delta / r)$. Consider an interval $\left[t_{0}, t_{0}+\right.$ $T$ ]. Since a queue can decrease by at most $M$ in a slot and $\left.X_{j}\left(t_{0}\right)>\overline{(} D\right)$ for each $j \in B, X_{j}(t)>D^{\prime}$ for each $t \in\left(t_{0}, t_{0}+T\right]$ for each $j \in B$. Let $X_{j}(t) \leq D^{\prime}$ for each $t \in\left(t_{0}, t_{0}+T\right]$ and $j \in Z \backslash B$. Then, since $L_{0}>\bar{D}>D^{\prime}$, no queue in $Z \backslash B$ is served in any slot in $[t, t+T]$ unless all queues in $B$ are disconnected in the slot. Thus, from (1) and $(2), \sum_{j \in Z \backslash B} X_{j}(t)$ increases by at least $r T-2 \Delta$ during $(t, t+T]$. Thus, at leat one queue in $Z \backslash B$ increases by at least $(r T-2 \Delta) / n$ during $(t, t+T]$. Note that $T$ is such that this increase exceeds $D^{\prime}$. Thus, $X_{j}(t)>D^{\prime}$ for some $t \in\left(t_{0}, t_{0}+T\right]$ and $j \in Z \backslash B$. The induction hypothesis now holds with $t_{1}$ being the first such time in $\left(t_{0}, t_{0}+T\right]$ where the above happens.

\section{Proof of Lemma 4}

Proof: We first introduce a few terminologies. Let $\mathcal{P}$ be the set of partitions of $Z$. For each partition $P=\left\{\hat{Q}_{1}, \hat{Q}_{2}, \ldots\right\} \in \mathcal{P}$, let $\mu_{i}^{P}=\mu\left(\hat{Q}_{i}, \overline{\left(\cup_{l \geq i+1} \hat{Q}_{l}\right)}\right)$, and $\rho_{i}^{P}=\sum_{j \in \hat{Q}_{i}} a_{j}$. Using Lemma 1 , we have $\sum_{1}^{i} \mu_{l}^{P}<$ $\sum_{1}^{i} \rho_{l}^{P}$ for each $i, P \in \mathcal{P}$. Consider some $\alpha \in(0,1)$ such that $\sum_{1}^{i} \mu_{l}^{P}<\sum_{1}^{i} \rho_{l}^{P}$ for each $i, P \in \mathcal{P}$. Let $u=\min _{P \in \mathcal{P}} \min _{i}\left(\sum_{1}^{i} \alpha \rho_{l}^{P}-\sum_{1}^{i} \mu_{l}^{P}\right)$. Clearly, $u>0$.

Consider some $\delta>0$. Now let $T$ be a positive integer such that $-u T+n \Delta<-\delta$.

Let $D_{\min }$ be a large enough number such that

$$
\begin{gathered}
\left(\frac{\frac{\left(\sqrt{D_{\min }}-M T\right)^{2}}{n}-M T}{\frac{\left(\sqrt{D_{\min }}-M T\right)^{2}}{n}+(2 n+1) M T}\right)^{3}>\alpha \\
\text { and } \sqrt{\frac{\left(\sqrt{D_{\min }}-M T\right)^{2}}{n}}>M T .
\end{gathered}
$$

Let $\mu=\sum_{i \in Z} \mu(\{i\}, \bar{\emptyset})$.

Let $D_{\max }$ be a large enough number such that

$$
\begin{aligned}
& D_{\max }>n \sqrt{\bar{D}}+2 n M T \\
& \text { and } \frac{-\delta}{(n \sqrt{\bar{D}}-M T)^{3}}+\frac{\mu T+\Delta}{\left(D_{\max }-M T\right)^{3}}<0 .
\end{aligned}
$$

Let $L_{\bar{D}, T}=D_{\max }+2(n+1) M T$. Thus, when $L>L_{\bar{D}, T}$, if a queue is below $D_{\max }+2 n M T$ at time $t$, it remains below $L$ in time interval $[t, t+T]$, and is served under longest connected queue policy in $[t, t+T]$.

Let $\hat{D}=\frac{(\sqrt{\bar{D}}-M T)^{2}}{n}$, and $D_{0}=n \sqrt{\bar{D}}$.

Now, the bounds on $\sum_{j \in Z} X_{j}^{-2}(t)$ in the statement of the lemma imply that $1 / \bar{D}<\sum_{j \in Z} X_{j}^{-2}(t)<1 / \hat{D}$. From the left hand side inequality, we have

$$
X_{j}(t)<D_{0} \quad \text { for at least one } j \text { in } Z,
$$

while since $\bar{D} \geq D_{\min }$, (16) and the right hand side inequality imply that $\min _{j \in Z} X_{j}(t) \geq \sqrt{\hat{D}}>M T$.

We now consider a specific partition of $Z\left\{Q_{1}, Q_{2}, \cdots, Q_{K}\right\}$ which is constructed as follows. Sort queues in $Z$ in nondecreasing order of their queue lengths and do the following.
1. Set $i=0$.

2 . Increment $i$ by one.

3. Move along the sorted list. As long as the difference in length between consecutive queues is less than or equal to $2 M T$, put every queue in $Q_{i}$.

4. If the next queue has length less than $D_{\max }$ return to (2). Else put the remaining queues in $Q_{i+1}$ (which may be an empty set) and terminate.

The above algorithm partitions $Z$. Let $D_{i}=$ $\min _{j \in Q_{i}} X_{j}(t)$ and $\left|Q_{i}\right|=n_{i}$. Note that (17) implies that $D_{\max }>D_{0}+2 n M T$, which leads to the following properties of the partition.

1. Subsets $Q_{1}, Q_{2}, \cdots, Q_{K-1}$ are all nonempty.

2. $\min _{j \in Q_{K}} X_{j}(t) \geq D_{\max }$.

3. $\max _{j \in Q_{i}} X_{j}(t)<\min _{l \in Q_{i+1}} X_{l}(t)-2 M T$ for $i=$ $1,2, \cdots, K-1$.

4. $\max _{j \in Q_{i}} X_{j}(t) \leq D_{i}+2 n M T$ for $i=1,2, \cdots, K-1$. 5. $D_{K-1}<D_{\max }$.

We now mention some properties of the above partition which we will use throughout the proof. Properties (4) and (5) and the fact that $L>L_{\bar{D}, T}=D_{\max }+2(n+1) M T$, ensure that all queues in $Q_{1}, \ldots, Q_{K-1}$ are served as per LCQ policy during $[t, t+T]$. Property (3) ensures that $\min _{j \in Q_{i+1}} X_{j}\left(t_{1}\right)>\max _{j \in Q_{i}} X_{j}\left(t_{1}\right)$ for each $i=1, \ldots, K-$ $1, t_{1} \in[t, t+T]$. Thus, a necessary condition for a queue in $Q_{i}$ to be served in a slot in $[t, t+T]$ is that at least one queue in $Q_{i}$ is connected and all queues in $\left(\cup_{l=1}^{i-1} Q_{l} \cup Z\right)$ are disconnected for $i=1, \ldots K-1$. Let $\mu_{i}=\mu\left(Q_{i}, \overline{\cup_{l=i+1}^{K} Q_{l}}\right)$, and $\rho_{i}=\sum_{j \in Q_{i}} a_{j}$. Note that $\left\{\mu_{i}\right\} \mathrm{s}$ and $\left\{\rho_{i}\right\} \mathrm{s}$ are $\left\{\mu_{i}^{P}\right\} \mathrm{s}\left\{\rho_{i}^{P}\right\} \mathrm{s}$ for partition $P=\left\{Q_{1}, \ldots, Q_{K}\right\}$. Clearly, $\mu \geq \max _{i} \mu_{i}$. Thus, from (1), $\sum_{j \in Q_{i}} S_{\{j\}}(t, t+T) \leq \mu_{i} T+\Delta$. Now,

$$
\begin{aligned}
& \sum_{j \in Z} X_{j}^{-2}(t+T)-\sum_{j \in Z} X_{j}^{-2}(t) \\
= & -\sum_{j \in Z}\left(X_{j}^{-1}(t+T)-X_{j}^{-1}(t)\right)^{2} \\
& +2 \sum_{j \in Z} X_{j}^{-1}(t+T)\left(X_{j}^{-1}(t+T)-X_{j}^{-1}(t)\right) \\
\leq & 2 \sum_{j \in Z} X_{j}^{-2}(t+T) X_{j}^{-1}(t)\left(X_{j}(t)-X_{j}(t+T)\right) \\
= & 2 \sum_{j \in Z} X_{j}^{-2}(t+T) X_{j}^{-1}(t) S_{\{j\}}(t, t+T) \\
& -2 \sum_{j \in Z} X_{j}^{-2}(t+T) X_{j}^{-1}(t) A_{\{j\}}(t, t+T) . \\
\leq & \sum_{j \in Q_{i}} \frac{S_{\{j\}}(t, t+T)}{X_{j}(t) X_{j}^{2}(t+T)} \frac{S_{\{j\}}(t, t+T)}{\left(X_{j}(t)-M T\right)^{3}}\left(\text { since } \min _{j \in Z} X_{j}(t)>M T\right), \\
\leq & \frac{1}{\left(D_{i}-M T\right)^{3}} \sum_{j \in Q_{i}} S_{\{j\}}(t, t+T) \\
\leq & \frac{\mu_{i} T+\Delta}{\left(D_{i}-M T\right)^{3}} \text { for } i=1, \cdots, K .
\end{aligned}
$$


The last inequality follows since $\sum_{j \in Q_{i}} S_{\{j\}}(t, t+T) \leq$ $\left.\mu_{i} T+\Delta\right)$. Since $\min _{j \in Z} X_{j}(t)>M T D_{j}>M T$ for each $j$. Thus, from property (2), we have

$$
\begin{aligned}
& \sum_{j \in Z} \frac{S_{\{j\}}(t, t+T)}{X_{j}(t) X_{j}^{2}(t+T)} \leq \sum_{i=1}^{K-1} \frac{\mu_{i} T+\Delta}{\left(D_{i}-M T\right)^{3}} \\
& +\frac{\mu_{K} T+\Delta}{\left(D_{\max }-M T\right)^{3}} \\
\geq & \sum_{j \in Q_{i}} \frac{A_{\{j\}}(t, t+T)}{X_{j}(t) X_{j}^{2}(t+T)} \frac{A_{\{j\}}(t, t+T)}{\left(X_{j}(t)+M T\right)^{3}} \\
\geq & \left.\frac{\sum_{j \in Q_{i}} A_{\{j\}}(t, t+T)}{\left(D_{i}+2 n M T+M T\right)^{3}} \text { (from property }(4)\right), \\
\geq & \frac{\sum_{j \in Q_{i}} a_{i} T-\Delta}{\left(D_{i}+(2 n+1) M T\right)^{3}} \quad \text { for } i=1, \cdots, K-1 .
\end{aligned}
$$

The last inequality follows from (2). Thus,

$$
\sum_{j \in Z} \frac{A_{\{j\}}(t, t+T)}{X_{j}(t) X_{j}^{2}(t+T)} \geq \sum_{i=1}^{K-1} \frac{\rho_{i} T-\Delta}{\left(D_{i}+(2 n+1) M T\right)^{3}}
$$

Since $\bar{D} \geq D_{\min }$ and due to (15),

$$
\left(\frac{\sqrt{\hat{D}}-M T}{\sqrt{\hat{D}}+(2 n+1) M T}\right)^{3}>\alpha
$$

Now, since $D_{i} \geq \min _{j \in Z} X_{j}(t) \geq \sqrt{\hat{D}}$, for each $i$,

$$
\left(\frac{D_{i}-M T}{D_{i}+(2 n+1) M T}\right)^{3}>\alpha \quad \text { for } \quad i=1,2, \cdots, K-1,
$$

which gives

$$
\begin{aligned}
\frac{\rho_{i}}{\left(D_{i}+(2 n+1) M T\right)^{3}}- & \frac{\mu_{i}}{\left(D_{i}-M T\right)^{3}} \geq \frac{\alpha \rho_{i}-\mu_{i}}{\left(D_{i}-M T\right)^{3}} \\
& \text { for } \quad i=1,2, \cdots, K-1 .
\end{aligned}
$$

From (21), (22), and (23) we have

$$
\begin{aligned}
\sum_{j \in Z} X_{j}^{-2}(t+T)-\sum_{j \in Z} X_{j}^{-2}(t) & \\
\leq & -T \sum_{i=1}^{K-1} \frac{\alpha \rho_{i}-\mu_{i}}{\left(D_{i}-M T\right)^{3}}+\frac{n \Delta}{\left(D_{1}-M T\right)^{3}} \\
& +\frac{\mu_{K} T+\Delta}{\left(D_{\max }-M T\right)^{3}} .
\end{aligned}
$$

We now lower bound $\sum_{i=1}^{K-1} \frac{\alpha \rho_{i}-\mu_{i}}{\left(D_{i}-M T\right)^{3}}$. Start with the right most term. If $\alpha \rho_{K-1} \geq \mu_{K-1}$, then the term $\frac{\alpha \rho_{K-1}-\mu_{K-1}}{\left(D_{K-1}-M T\right)^{3}}$ is positive and the sum can be lower bounded by $\sum_{i=1}^{K-2} \frac{\alpha \rho_{i}-\mu_{i}}{\left(D_{i}-M T\right)^{3}}$. If, on the other hand, $\alpha \rho_{K-1}<\mu_{K-1}, \frac{\alpha \rho_{K-1}-\mu_{K-1}}{\left(D_{K-1}-M T\right)^{3}} \geq \frac{\alpha \rho_{K-1}-\mu_{K-1}}{\left(D_{K-2}-M T\right)^{3}}$, and the sum can be lower bounded by $\sum_{i=1}^{K-3} \frac{\alpha \rho_{i}-\mu_{i}}{\left(D_{i}-M T\right)^{3}}+$ $\frac{\alpha\left(\rho_{K-2}+\rho_{K-1}\right)-\left(\mu_{K-2}+\mu_{K-1}\right)}{\left(D_{K-2}-M T\right)^{3}}$. Continuing in the same manner we obtain, $\sum_{i=1}^{K-1} \frac{\alpha \rho_{i}-\mu_{i}}{\left(D_{i}-M T\right)^{3}} \geq \frac{\sum_{i=1}^{l} \alpha \rho_{i}-\mu_{i}}{\left(D_{1}-M T\right)^{3}}$ for some $l \in\{1,2, \cdots, K-1\}$. The second term can be lower bounded by $u /\left(D_{1}-M T\right)^{3}$. Thus, from (24) we have

$$
\begin{aligned}
\sum_{j \in Z} X_{j}^{-2}(t+T)-\sum_{j \in Z} X_{j}^{-2}(t) \leq & \frac{-u T+n \Delta}{\left(D_{1}-M T\right)^{3}} \\
& +\frac{\mu_{K} T+\Delta}{\left(D_{\max }-M T\right)^{3}} .
\end{aligned}
$$

Now, since $-u T+n \Delta<-\delta$, where $\delta>0$, and $\mu_{K} \leq \mu$, from (19), we have

$$
\begin{aligned}
\sum_{j \in Z} X_{j}^{-2}(t+T)-\sum_{j \in Z} X_{j}^{-2}(t) \leq & \frac{-\delta}{\left(D_{0}-M T\right)^{3}} \\
& +\frac{\mu T+\Delta}{\left(D_{\max }-M T\right)^{3}} .
\end{aligned}
$$

From (18), (25) is negative and the lemma follows.

\section{REFERENCES}

[1] A. Aaram, M.H.R. Khouzani, S. Sarkar, and L. Tassiulas. A fair scheduling policy for wireless channels with intermittent connectivity. Technical report, Univ. of Pennsylvania, Philadelphia, PA, March 2008. http://www.seas.upenn.edu/ swati/publication.htm.

[2] P. Chaporkar, K. Kar, and S. Sarkar. Throughput guarantees through maximal scheduling in multihop wireless networks. In Proceedings of $43 d$ Annual Allerton Conference on Communication, Control and Computing, Allerton, Monticello, Illinois, September 28-30 2005.

[3] X. Lin and N. Shroff. The impact of imperfect scheduling on crosslayer rate control in multihop wireless networks. In Proceedings of INFOCOM, Miami, FL, Mar 2005.

[4] M. Neely, E. Modiano, and C. Li. Fairness and optimal stochastic control for heterogeneous networks. In Proceedings of INFOCOM, Miami, Florida, March 2005.

[5] S. Ray and S. Sarkar. Arbitrary throughput versus complexity tradeoffs in wireless networks using graph partitioning. In Proceedings of Information Theory and Applications Second Workshop, University of California at San Diego, 2007.

[6] S. Sarkar and L. Tassiulas. End-to-end bandwidth guarantees through fair local spectrum share in wireless ad-hoc networks. IEEE Transactions on Automatic Control, September 2005.

[7] L. Tassiulas. Adaptive back-pressure congestion control based on local in formation. IEEE Transactions on Automatic Control, February 1995

[8] L. Tassiulas and A. Ephremidis. Stability properties of constrained queueing systems and scheduling policies for maximum throughput in multihop radio networks. IEEE Transactions on Automatic Control, 37(12):1936-1948, Dec 1992.

[9] X. Wu and R. Srikant. Regulated maximal matching: a distributed scheduling algorithm for multihop wireless networks with node-exclusive spectrum sharing. In Proceedings of IEEE CDCECC'05, Seville, Spain, Dec 2005. 